\title{
EFECTIVIDAD DEL PROGRAMA “COME LO NUESTRO" EN LOS CONOCIMIENTOS, ACTITUDES Y PRÁCTICAS SOBRE EL CONSUMO DE QUINUA, CAÑIHUA Y KIWICHA EN ALUMNOS DEL SEXTO GRADO DE PRIMARIA Y SUS FAMILIARES DEL COLEGIO ADVENTISTA DEL TITICACA, PUNO, PERÚ, 2007
}

\author{
Manuel, CONCHA TOLEDO ${ }^{1}$
}

\begin{abstract}
RESUMEN
Objetivos: Determinar la efectividad del programa “Come lo Nuestro" en los conocimientos, actitudes y prácticas sobre el consumo de quinua, cañihua y kiwicha en alumnos del sexto grado de primaria y sus familiares del Colegio Adventista del Titicaca, Puno, (Perú), 2007. Metodología: Se aplicó un pretest a 24 alumnos del sexto grado de primaria del Colegio Adventista del Titicaca: 10 preguntas de conocimientos, 13 de actitud y 6 de prácticas; un pre test a los familiares de los mismos alumnos: 10 preguntas de conocimientos, 19 de actitud y 9 de prácticas. Se aplicó un programa educativo promocional que consistió en el empleo de dos módulos: uno acerca de los conocimientos sobre el valor nutritivo de la quinua, cañihua y kiwicha y otro de motivación para el cambio conductual. Ambos módulos se desarrollaron por espacio de 9 semanas; al final de las cuales se aplicó un postest a ambos grupos. Resultados: Los resultados señalaron que el nivel de conocimientos fue mayor al final de la intervención, la actitud fue positiva y las prácticas de consumo se incrementaron. Conclusión: El programa "Come lo Nuestro" tuvo un impacto positivo en los conocimientos, actitudes y prácticas sobre el consumo de quinua, cañihua y kiwicha, en alumnos del sexto grado de primaria y sus familiares del Colegio Adventista del Titicaca.
\end{abstract}

Palabras clave: Modelo PRECEDE-PROCEDE, quinua, cañihua, kiwicha, conocimientos, actitud y prácticas.

\begin{abstract}
Objectives: To determine the effectiveness of the program "Eat What is Ours" in knowledge, attitudes and practices on the consumption of quinoa, cañihua, and amaranth in sixth grade students and their families at the Lake Titicaca Adventist Elementary School, Puno (Peru), 2007. Methodology: A pre-test was applied to 24 students in sixth grade at the Lake Titicaca Adventist Elementary School: 10 knowledge questions, 13 about attitude, and 6 about practice; a pre-test was applied to the families of these students: 10 knowledge questions, 19 about attitude, and 9 about practices. A promotional educational program was applied which consisted in the use of two modules: one on knowledge about the nutritional value of quinoa, cañihua, amaranth and another about motivation for behavior change. Both modules were developed in 9 weeks at the end of which a post test was applied to both groups. Results: The results showed that the level of knowledge was higher at the end of the intervention; the attitude was positive and increased consumption practices. Conclusions: The "Eat What is Ours" program had a positive impact on knowledge, attitudes and practices on the consumption of quinoa, cañihua, and amaranth in students from sixth grade and their families at the Lake Titicaca Adventist Elementary School.
\end{abstract}

Key Words: PRECEDE-PROCEED model, quinoa, cañihua, amaranth, knowledge, attitude and practices.

\footnotetext{
${ }^{1}$ Químico Farmacéutico, Magíster en Salud Pública, Secretario Académico y Docente de la Facultad de Ciencias de la Salud de la Universidad Peruana Unión
} 


\section{INTRODUCCIÓN}

La nutrición es un factor importante para la conservación de la salud, prevenir enfermedades y evitar la desnutrición crónica en la población, ya que la educación promueve cambios en los conocimientos, actitudes y prácticas de las personas. El modelo de Lawrence Green y Marshall Kreuter, PRECEDE-PROCEDE, (Green, L. y Kreuter, M.) utilizado en esta investigación permite planear y evaluar un programa de intervención como parte de las actividades de educación y promoción de la salud. Es de especial relevancia para la salud de la población aumentar el grado de conocimientos, actitudes y prácticas sobre el consumo de quinua, cañihua y kiwicha.

La alimentación constituye uno de los componentes esenciales del bienestar y un valioso indicador de los niveles de vida de una comunidad y representa, junto con otros indicadores, el grado de desarrollo de un país (Collazos, C 1993).

En el período 2001- 2003, según estimaciones de la FAO, había 854 millones de personas subnutridas a escala mundial (ONU, 2006).

En el Perú, la situación de la inseguridad alimentaria empeoró durante el decenio de 1970 y especialmente en el de 1980 (ONU, 2006). Sin embargo, estudiantes de primaria, de 6 a 9 años de edad, presentaron altas tasas de desnutrición crónica en el año 2005 (Educatos, 2006).

Datos estadísticos actuales refieren que el 32\% del grupo etario de 6 a 11 años de edad presenta anemia ferropénica y en el grupo de 12 a 17 años, un 35\% (INEI, 2008).

La desnutrición crónica en la infancia temprana afecta la capacidad intelectual de los individuos (Berkman, 2002), desarrollo físico, patrones de comportamiento durante la adultez, riesgos de enfermar y morir (Pollit, 1990); así como la reducción de la capacidad productiva durante la adultez (Norgan, 2000).

En la sierra, los escasos recursos económicos de sus habitantes afecta directamente a la población infantil; por otra parte se sabe que el consumo de granos andinos es muy bajo, con un consumo per cápita de 0,417 Kg./año (CONDESAN, 2000).

No se han encontrado estudios que evalúen la efectividad de programas educativos dirigidos hacia la educación del consumo de alimentos de origen andino en la ciudad de Juliaca.
Según reporte oral de los docentes del Colegio Adventista del Titicaca, de los padres de familia y de los propios alumnos no consumen frecuentemente productos de origen andino tales como quinua, kiwicha y cañihua.

Este contexto motivó al investigador realizar el presente trabajo con el objetivo de determinar la efectividad del programa "Come lo Nuestro" en los conocimientos, actitudes y prácticas sobre el consumo de quinua, cañihua y kiwicha en alumnos del sexto grado de primaria y sus familiares del Colegio Adventista del Titicaca, Puno, (Perú), 2007.

\section{MATERIAL Y MÉTODOS}

El programa de intervención "Come lo Nuestro" estuvo bajo la coordinación general del investigador, quien requirió la colaboración de ocho estudiantes del segundo año de enfermería para la aplicación del programa, a quienes se les capacitó por espacio de tres semanas. El programa tuvo una duración de 9 semanas, empezando el 23 de setiembre y terminó el 2 de diciembre del 2007. Se desarrolló íntegramente en las instalaciones del Colegio Adventista del Titicaca.

Se emplearon tres teorías de cambio conductual: Teoría transteorética de cambios de comportamiento a través del conocimiento impartido, toma de decisiones y el uso de reforzadores; teoría del aprendizaje social y la teoría de la acción razonada.

Se emplearon dos módulos: uno acerca de los conocimientos sobre el valor nutritivo de la quinua, cañihua y kiwicha, asimismo otro de motivación para el cambio conductual.

Implementación de talleres teórico-prácticos desarrollados en grupos de ocho personas para la preparación de las distintas recetas en base a quinua, cañihua y kiwicha.

Se aplicó el test de conocimientos, actitudes y prácticas en relación al consumo de quinua, cañihua y kiwicha antes y después del programa de intervención, se aplicó asimismo el programa de intervención "Come lo Nuestro” siguiendo el cronograma establecido.

Se emplearon los siguientes instrumentos y técnicas:

1. Test para identificar el nivel de conocimientos, actitudes y prácticas sobre el consumo de alimentos de origen andino.

2. Hoja de control diario para alumnos.

3. Autorreporte semanal de madres para verificar la frecuencia de preparación y formas de consumo de alimentos de origen andino. 
El test estuvo organizado de la siguiente manera: objetivo, instrucciones, datos generales, conocimientos, actitudes y prácticas sobre el consumo de quinua, cañihua y kiwicha. Se aplicó antes y después del programa de intervención “Come lo Nuestro".

El procesamiento se efectuó utilizando el software estadístico SPSS 15.0, los datos obtenidos fueron vaciados a la matriz de datos para el análisis respectivo.

Para verificar las hipótesis planteadas, se empleó la prueba T de Student de comparación de medias. Para medir la validez del instrumento se empleó el juicio de expertos. Para medir la confiabilidad del instrumento se utilizó la prueba de Coeficiente Alpha de Cronbach.

\section{RESULTADOS}

Tabla 1. Alumnos y familiares del sexto grado de primaria del Colegio Adventista del Titicaca, según el grado de conocimientos sobre el consumo de quinua, cañihua y kiwicha antes y después del programa “Come lo Nuestro”, Juliaca 2007

\begin{tabular}{|c|c|c|c|c|c|c|c|c|}
\hline \multirow{3}{*}{$\begin{array}{l}\text { NIVEL DE } \\
\text { CONOCIMIENTO } \\
\text { GENERAL }\end{array}$} & \multicolumn{4}{|c|}{ ALUMNOS } & \multicolumn{4}{|c|}{ FAMILIARES } \\
\hline & \multicolumn{2}{|c|}{ PRE TEST } & \multicolumn{2}{|c|}{ POST TEST } & \multicolumn{2}{|c|}{ PRE TEST } & \multicolumn{2}{|c|}{ POST TEST } \\
\hline & $\mathrm{n}$ & $\%$ & $\mathrm{n}$ & $\%$ & $\mathrm{n}$ & $\%$ & $\mathrm{n}$ & $\%$ \\
\hline Conocimiento pésimo & 0 & 0,0 & 0 & 0 & 0 & 0,0 & 0,0 & 0,0 \\
\hline Conocimiento bajo & 1 & 4,2 & 0 & 0 & 1 & 4,2 & 0 & 0,0 \\
\hline Conocimiento regular & 5 & 20,8 & 1 & 4,2 & 7 & 29,2 & 0 & 0,0 \\
\hline Conocimiento bueno & 18 & 75,0 & 5 & 20,8 & 16 & 66,7 & 14 & 62,5 \\
\hline Conocimiento excelente & 0 & 0,0 & 18 & 75,0 & 0 & 0,0 & 9 & 37,5 \\
\hline TOTAL & 24 & 100 & 24 & 100 & 24 & 100 & 24 & 100 \\
\hline
\end{tabular}

Fuente: Encuesta de conocimientos, actitudes y prácticas aplicada a estudiantes del $6^{\circ}$ to grado de primaria del Colegio Adventista del Titicaca, Juliaca 2007.

En cuanto al grado de conocimientos, en la tabla 1 se puede apreciar que antes de aplicar el programa "Come lo Nuestro", el 75,0\% de los alumnos del sexto grado de primaria del Colegio Adventista del Titicaca, obtuvo un grado de conocimientos bueno sobre consumo de quinua, cañihua y kiwicha, el 20, 8\% obtuvo un grado de conocimientos regular y sólo el 4,2\% un grado de conocimientos bajo. Sin embargo, después de aplicar el programa "Come lo Nuestro" el grado de conocimientos mejoró, el75,9\% presentó un grado de conocimientos excelente, el 20,8\% mostró un grado de conocimientos bueno y sólo el 4,2\% un grado de conocimientos de los familiares de los alumnos del sexto grado de primaria del Colegio Adventista del Titicaca antes de aplicar el programa, el 66,7\% reveló un grado de conocimientos bueno sobre consumo de quinua, cañihua y kiwicha; el 29,2\% obtuvo un grado de conocimientos regular y sólo el 4,2\% un grado de conocimientos bajo. Sin embargo, después de aplicar el programa "Come lo Nuestro" el grado de conocimientos mejoró el 62,5\% obtuvo un grado de conocimientos bueno y el 47,5\% mostró un grado de conocimientos excelente sobre consumo de quinua, cañihua y kiwicha.

Tabla 2. Alumnos y familiares del sexto grado de primaria del Colegio Adventista del Titicaca, según las actitudes en relación al consumo de quinua, cañihua y kiwicha antes y después del programa “Come lo Nuestro”, Juliaca 2007.

\begin{tabular}{|cccccccccc} 
& \multicolumn{4}{c}{ ALUMNOS } & \multicolumn{4}{c}{ FAMILIARES } \\
ACTITUD GENERAL & \multicolumn{3}{c}{ PRE TEST } & \multicolumn{1}{c}{ POST TEST } & PRE TEST & POST TEST \\
& $\mathrm{n}$ & $\%$ & $\mathrm{n}$ & $\%$ & $\mathrm{n}$ & $\%$ & $\mathrm{n}$ & $\%$ \\
\hline Actitud negativa & 15 & 62,5 & 2 & 8,3 & 15 & 62,5 & 0 & 0,0 \\
Actitud positiva & 9 & 37,5 & 22 & 91,7 & 9 & 37,5 & 24 & 100 \\
\hline TOTAL & 24 & 100 & 24 & 100 & 24 & 100 & 24 & 100 \\
\hline
\end{tabular}

Fuente: Encuesta de conocimientos, actitudes y prácticas, aplicada a estudiantes del $6^{\circ}$ to grado de primaria del Colegio Adventista del Titicaca, Juliaca, 2007. 
En la tabla 2 se puede apreciar que antes de aplicar el programa "Come lo Nuestro", el 62,5\% de los alumnos del sexto grado de primaria del Colegio Adventista del Titicaca presentó actitud negativa y sólo el $37,5 \%$ presentó actitud positiva. Después de aplicar el programa "Come lo nuestro", los resultados cambiaron totalmente, pues el 91,7\% presentó actitud positiva y sólo el 8,3\% actitud negativa. Antes de aplicar el programa de intervención "Come lo Nuestro", el 62,5\% de los familiares de los alumnos del sexto grado de primaria del Colegio Adventista del Titicaca presentó actitud negativa y sólo el $37,5 \%$ presentó actitud positiva, después de aplicar el programa "Come lo Nuestro", los resultados totalmente, pues el $100 \%$ presentó actitud positiva.

Tabla 3. Alumnos y familiares del sexto grado de primaria del Colegio Adventista del Titicaca, según prácticas de consumo de quinua, cañihua y kiwicha antes y después del programa “Come lo Nuestro”, Juliaca 2007.

\begin{tabular}{|c|c|c|c|c|c|c|c|c|}
\hline \multirow{3}{*}{ PRÁCTICA GENERAL } & \multicolumn{4}{|c|}{ ALUMNOS } & \multicolumn{4}{|c|}{ FAMILIARES } \\
\hline & \multicolumn{2}{|c|}{ PRE TEST } & \multicolumn{2}{|c|}{ POST TEST } & \multicolumn{2}{|c|}{ PRE TEST } & \multicolumn{2}{|c|}{ POST TEST } \\
\hline & $\mathrm{n}$ & $\%$ & $\mathrm{n}$ & $\%$ & $\mathrm{n}$ & $\%$ & $\mathrm{n}$ & $\%$ \\
\hline Nunca & 1 & 4,2 & 0 & 0 & 2 & 8,3 & 0,0 & 0,0 \\
\hline A veces (1 vez por semana) & 10 & 41,7 & 3 & 12,5 & 11 & 45,8 & 0,0 & 0,0 \\
\hline Casi siempre (2 veces por semana) & 12 & 50,0 & 7 & 29,2 & 10 & 41,7 & 7 & 29,2 \\
\hline Frecuentemente (3 veces por semana) & 1 & 4,2 & 14 & 58,3 & 1 & 4,2 & 17 & 70,8 \\
\hline TOTAL & 24 & 100 & 24 & 100 & 24 & 100 & 24 & 100 \\
\hline
\end{tabular}

Fuente: Encuesta de conocimientos, actitudes y prácticas, aplicada a estudiantes del $6^{\circ}$ to grado de primaria del Colegio Adventista del Titicaca, Juliaca, 2007.

La tabla 3 muestra que antes de aplicar el programa de intervención "Come lo Nuestro", el 4,2\% de los alumnos del sexto grado de primaria del Colegio Adventista del Titicaca dijo no consumir quinua, cañihua o kiwicha; el 41,7\% dijo consumir una vez por semana, el 50,0\% dos veces por semana y sólo el 4,2\% dijo consumir tres veces por semana. Después de aplicar el programa de intervención "Come lo Nuestro", sólo el 12,5\% dijo consumir una vez por semana, el 29,2\% lo seguía haciendo dos veces por semana y el 58,3\% dijo consumir tres veces por semana. Antes de aplicar el programa de intervención "Come lo Nuestro", el 8,3\% de los familiares de los alumnos del sexto grado de primaria del Colegio Adventista del Titicaca dijo no consumir nunca quinua cañihua o kiwicha; el45,8\% sólo consumía una vez por semana, el 41,7\% dos veces por semana y sólo el 4,2\% consumía tres veces por semana; después de aplicar el programa de intervención "Come lo Nuestro", el 29,2\% consumía quinua, cañihua, o kiwicha dos veces por semana, y $70,8 \%$ tres veces por semana.

\section{DISCUSIÓN}

Según los resultados de la tabla №1 el mayor porcentaje de la muestra mostró un grado de conocimiento bueno y regular antes del programa de intervención "Come lo Nuestro. Sin embargo esto no significa que tengan un estilo de vida alimentario saludable, porque el conocimiento por sí solo no cambia a las personas. La OPS/OMN (2003), mencionan que el aumento de conocimientos no asegura un cambio conductual, pero es importante porque constituye el primer paso para el proceso de cambio. Marinof (2001) (citado por Chanducas, 2006), sostiene que la información es necesaria porque contribuye al cambio de actitudes. Jackson (2005) (citado por Chanducas, 2006), menciona que existe una relación entre conocimientos, actitudes y conductas, pero que esta relación es mínima. también se considera que la conducta está condicionada por aspectos cognitivos pues lo que sabemos y lo que pensamos afecta a la forma en que actuamos; por otro lado, el conocimiento es necesario, pero no suficiente para producir un cambio de conducta: las percepciones, la motivación, la habilidades y los factores sociales también juegan un papel fundamental.

Según la tabla $\mathrm{N}^{\circ} 2$ el mayor porcentaje presentó actitud negativa antes del programa de intervención "Come lo Nuestro", en relación al consumo de quinua, cañihua y kiwicha. Las actitudes, se adquieren durante el proceso de sociabilización, mediante el cual se interioriza lo que está bien o lo que está mal, lo que agrada o desagrada, lo que gusta o disgusta. Con el paso de los años, las actitudes se fortalecen porque los jóvenes son como gafas de colores que tiñen o filtran la información, dejan pasar con más facilidad aquella información que les agrada y rechazan o ignoran aquella que les desagrada (Dawes, R, 1983). Después del programa de intervención "Come lo Nuestro", el 91,7\% de los alumnos y el $100 \%$ de los familiares presentaron actitud positiva. 
En un estudio a nivel mundial, Fernández-Abascal (2001) (citado por Huaringa, 2006), resaltó la importancia que tienen las actitudes positivas en un estudio "Preferencia y gusto de los alimentos y lograr el consumo adecuado de ellos", "Las personas que disfrutan de la vida y potencian afectos positivos tendrán mejores pronósticos que las que no lo hagan”, apuntó. “La mayoría de estudios médicos señala que la actitud positiva tiene una especial trascendencia ya que mejoran el rendimiento y fortalecen la salud.

Según los resultados de la tabla $\mathrm{N}^{\circ} 3$, antes del programa de intervención "Come lo Nuestro", el 50,0\% de los alumnos refirieron consumir potajes a base de quinua, cañihua y kiwicha por lo menos dos veces por semana y el $41,7 \%$ de kiwicha por lo menos una vez por semana, el 45,8\% de los familiares también consumía quinua, cañihua y kiwicha con la misma frecuencia; el $4,2 \%$ de los alumnos y el 8,3\% de los familiares no consumía nunca. Estos datos concuerdan con estudios realizados por Ayala y Vilchez (1996), en la ciudad de Lima, donde un 23,1\% dijo consumir quinua por lo menos una vez por semana y $21,7 \%$ kiwicha. Este estudio no reportó consumo de cañihua.

Después de aplicar el programa de intervención “Come lo Nuestro", 58,3\% de los alumnos reportó que consumía alguno de estos alimentos por lo menos tres veces por semana, $70,8 \%$ de los familiares reportó que consumía alguno de estos alimentos con la misma frecuencia. Jiménez Menes, Fabiola (2005), menciona que una conducta se repite cuando es satisfactoria en algún as- pecto: a los sentidos, a las emociones, a los valores y a los principios, a la autoestima, al deseo de comodidad a las creencias religiosas o de otro tipo, a la interacción con los demás miembros del grupo familiar, comunal o nacional y a las relaciones con el entorno físico y económico. En otras palabras, el hábito debe ser coherente con el resto de la vida diaria y se conserva por ser agradable, cómodo o útil, ya sean estos tributos reales o aparentes y se perciban de manera consciente o no.

\section{CONCLUSIONES}

El programa "Come lo Nuestro" tuvo un impacto positivo en los conocimientos, actitudes y prácticas sobre el consumo de quinua, cañihua y kiwicha, en alumnos del sexto grado de primaria y sus familiares del Colegio Adventista del Titicaca.

\section{RECOMENCIONES}

Por lo buenos resultados obtenidos en el programa “Come lo Nuestro”, se recomienda:

1. Que sea adoptado como un programa permanente en el Colegio Adventista del Titicaca.

2. Aplicar el programa “Come lo Nuestro", por mayor periodo de tiempo que permita cambios en actitudes y prácticas de manera permanente.

3. Extender el programa "Come lo Nuestro", a todos los niveles del Colegio Adventista del Titicaca.

\section{REFERENCIAS BIBLIOGRÁFICAS}

1. Ayala, G. Aporte de los cultivos andinos a la nutrición humana. UNMSM. Lima, Perú; 2005.

2. Beaglehole, R., Bonita, R., Kjellstrom, T. Epidemiología básica. Publicación científica No 551 , OMS. Ginebra; 1994.

3. Berkman DS, Lescano AG, Gilman RH, Black MM and Lopez S. Stunting. Diarrheal disease and parasitic infections in peruvian infants: their effect on cognitive test scores in late childhood. Lancet; 2002. 359:564-71

4. Bonifacio, A.. El futuro de los productos andinos en la región alta y los valles centrales de los andes. Estudio de prospectiva para los productos del altiplano; 2006.
5. Collazos, C.. La composición de alimentos de mayor consumo en el Perú. 6ta edición. Ministerio de Salud. Instituto Nacional de Nutrición, Banco Central de Reserva, Lima;1993.

6. CONDESAN. Desarrollo socioeconómico en las tierras altas de los andes del Perú mediante una productividad agropecuaria más eficiente. Informe de progreso, mayo; 2000, 2000-99. CIRNMA-AECI-INIA-ESPAÑA-CIP/CONDESAN. Puno, Perú.

7. Chanducas, B.. Impacto del Programa "Mi Amigo el Hierro" en el estilo de vida alimentario para prevenir la anemia en los alumnos del 2do y 3er año de Nutrición Humana de la Universidad Peruana Unión; Lima, 2006. 
8. De Abdraca Oyarzun, I. Salas I.. Interacción madre-hijo y conducta del niño en preescolares con antecedentes de anemia por deficiencia de hierro en la infancia. Archivos Latinoamericanos de Nutrición; 1993. Vol. 43 No 3. Chile.

9. EDUCATOS. Ministerio de Educación. Magnitudes e indicadores de la educación peruana. Unidad de estadística educativa No 4, septiembre; 2006.

10. Ferroni, M.. La alimentación del campesinado andino: Mitos y realidad. Citado en: Agricultura y alimentación. Bases de un nuevo enfoque. Lajo, M. et al.; 1982.

11. Green, L. y Kreuter, M.. Health promotion planning. an educational and envoromental approach 2da edition. Mayfield: Publishing Company; 1991. INEI. Estado de la niñez en el Perú. UNICEF, resumen ejecutivo. On line: recuperado el 7 de agosto del 2008 de:http://www.unicef.org/peru/spanish/ Resumen_ENP_Abril_2008.pdf.

12. Macedo, G.A.. Consumption of quinoa in Peru. Food Reviews International; 2003 Vol.19, Issue $1 \& 2$.

13. Madruga Acerete, D.. Alimentación del adolescente. Protocolos diagnósticos y terapéuticos en pediatría. On line: recuperado el 7 de agosto del 2008 de: http://www.aeped.es/protocolos/nutricion/1.pdf
14. MINSA, Encuesta nacional de indicadores nutricionales, bioquímicos, socioeconómicos y culturales relacionados con las enfermedades crónicas degenerativas. Lima, Perú; 2006.

15. Mujica, A. \& Jacobsen, E.. La Quinua y sus parientes silvestres. Universidad Mayor de San Andrés, La Paz, Bolivia; 2006.

16. Mujica A. Jacobsen, E., Canahua, A. Ayala, G.. Proyecto: Fortalecimiento de la producción, comercialización y consumo de los cultivos andinos en zonas de extrema; 2006.

17. Norgan NG. Long-term physiological and economic consequences of growth retardation in children and adolescents. Proc Nutr Soc; 2000. 59(2):245-5

18. ONU. El estado de la seguridad alimentaria en el mundo. La erradicación del hambre en el mundo. Evaluación de la situación diez años después de la Cumbre Mundial sobre la Alimentación; 2006.

19. Pamplona, R. J.. Nuevo estilo de vida disfrútalo. Alimentos que curan y previenen. Editorial Safeliz. Segunda Edición. Buenos Aires - Argentina; 1998.

20. Pollit, D.. Desnutrición e infección en los salones de clase. París. UNESCO; 1990.

21. Zapata Acha, S.. Posibilidades y potencialidad de la agroindustria en el Perú en base a la biodiversidad y los bionegocios; 2001. 